\title{
Treatment of Bothrops alternatus envenomation by Curcuma longa and Calendula officinalis extracts and ar-turmerone
}

[Tratamento local do envenenamento por Bothrops alternatus com extrato de Curcuma longa e Calendula officinalis e ar-turmerone]

\author{
M.M. Melo ${ }^{1}$, G.G. Habermehl ${ }^{2}$, N.J.F. Oliveira ${ }^{1}$, E.F. Nascimento ${ }^{1}$, M.M.B. Santos ${ }^{1}$, M. Lúcia $^{3}$ \\ ${ }^{1}$ Escola de Veterinária - Universidade Federal de Minas Gerais \\ Caixa Postal 567 \\ 30123-970 - Belo Horizonte, MG \\ ${ }^{2}$ Chemisches Institut, Tierärztliche Hochschule, Hannover, Germany \\ ${ }^{3}$ Departamento de Biologia - UNIFENAS - Alfenas, MG
}

\begin{abstract}
It was investigated the efficiency of two extracts of plants and one fraction of their properties against the local effects of bothropic envenomation. Bothrops alternatus venom $(1.25 \mu \mathrm{g})$ diluted in $100 \mu \mathrm{l}$ of sterile saline solution was inoculated (intradermally) into the shaved dorsal back skin of 30 New Zealand rabbits. The animals were divided in six groups receiving the following treatments: group I: subcutaneous application of Curcuma longa extract (1.0ml); group II: topic treatment of Curcuma longa hydroalcoholic extract $(1.0 \mathrm{ml})$; group III: topic application of ar-turmerone in vaseline (1.0g); group IV: topic application of Curcuma longa methanolic extract $(1.0 \mathrm{ml})$; group V: topic application of Calendula officinalis ointment $(1.0 \mathrm{~g})$; group VI: topic application of saline $(1.0 \mathrm{ml})$. These treatments were done at 30 minutes, and at 2, 4, 24 and 72 hours after venom inoculation. Intensity of local edema, hemorrhagic halo and necrosis were evaluated until $168 \mathrm{~h}$ after that. Additionally, seven days after the Bothrops venom inoculation, blood was collected from heart with and without EDTA (10\%) for hemogram and biochemical parameters (total protein, blood urea nitrogen, creatinine, and fibrinogen) and all the animals were anesthetized, sacrificed by ether inhalation and submitted to necropsy. Fragments of tissues were taken for histopathological evaluation. The most efficient treatment for inhibition of edema, necrosis and local hemorrhage after Bothrops alternatus venom was the topic application of ar-turmerone.
\end{abstract}

Keywords: plant extract, ar-turmerone, Bothrops alternatus, rabbit

\section{RESUMO}

Investigou-se a eficácia do extrato de plantas no tratamento local do envenenamento botrópico. Veneno de serpentes Bothrops alternatus $(1,25 \mu \mathrm{g}$ ) diluído em $100 \mu \mathrm{l}$ de solução salina estéril foi inoculado (via intradérmica) entre as escápulas de 30 coelhos. Os animais foram divididos em seis grupos (tratamentos): grupo I: tratamento subcutâneo com extrato de Curcuma longa; grupo II: tratamento tópico com extrato hidroalcoólico de Curcuma longa; grupo III: tratamento tópico com ar-turmerone em vaselina; grupo IV: tratamento tópico com extrato metanólico de Curcuma longa; grupo V: tratamento tópico com pomada de Calendula officinalis e grupo VI: aplicação tópica de solução salina a 0,9\% (Controle). Os tratamentos foram realizados 30 minutos, $2 \mathrm{~h}, 4 \mathrm{~h}, 24 \mathrm{~h}$ e $72 \mathrm{~h}$ após a inoculação do veneno. Foram avaliados intensidade de edema local (com paquímetro), halo hemorrágico (régua com gabaritos circulares) e presença de necrose. Sete dias após a inoculação do veneno botrópico (168h) foi coletado sangue do coração, com e sem EDTA, para realização de hemograma, dosagem plasmática de fibrinogênio e dosagens séricas de proteina total, uréia e creatinina. Após as coletas de sangue, todos os

Recebido para publicação em 3 de outubro de 2003

Recebido para publicação, após modificações, em 1 de setembro de 2004

E-mail: marilia@vet.ufmg.br 
animais foram anestesiados, sacrificados com inalação pelo éter etílico e necropsiados. Fragmentos de pele foram retirados para avaliação histopatológica. Os resultados obtidos mostraram que o tratamento mais eficaz para inibição da evolução do edema, necrose e hemorragia local após envenenamento com Bothrops alternatus foi a aplicação tópica de ar-turmerone.

Palavras-chave: extrato de planta, ar-turmerone, Bothrops alternatus, coelho

\section{INTRODUCTION}

Envenomation caused by Bothrops genus snakes induces severe and rapid onset of local tissue damage. This effect is characterized by pain, edema, hemorrhage and myonecrosis (Rosenfeld, 1971). Local edema is a typical manifestation of Bothrops bites. It is probably caused by a direct effect of venom on vessels by metalloproteinases (Chaves et al., 1995) and by releasing of endogenous mediators like histamine, kinins and prostaglandins, due to the action of venom components on mast cells, kininogens and phospholipids, respectively (Gutiérrez and Lomonte, 1989; Moura-da-Silva et al., 1996). Systemic and local bleeding are complications commonly found in viperine envenoming. In Bothrops snake venoms, which also possess procoagulants toxins, bleeding may be particularly severe because fibrinogen consumption, resulting in not coagulable blood, may coexist with thrombocitopenia. The action of haemorrhagins on the vessel contributes to a potentially life-threatening haemorrhage (Kamiguti et al., 1996). Muscle necrosis is due to the action of myotoxins, some of which have phospholipase $\mathrm{A}_{2}$ structure. Myotoxins affect the integrity of the skeletal muscle plasma membrane by a mechanism not directly related to phospholipid hydrolysis. In addition, in the muscle may occur ischemia as a consequence of drastic vasculature damage (Gutiérrez and Lomonte, 1989).

Man has successfully used plants against numerous diseases caused by different pathological agents. Among the plants worldwide used in medicine, several species have been shown to possess anti-snake venom activity, protecting humans and animals against snake bite (Mors et al., 2000). Curcuma longa and Calendula officinalis belong to that group of plants. Several substances, among them curcumine and ar-turmerone, have been earlier isolated from rhizomes of Curcuma longa (Ferreira et al., 1992a). Many flavonoids with anti-inflammatory activity were found in high amounts in Calendula officinalis. Triterpene saponins and carotenoids may also be important (Mors et al., 2000).

The only acceptable treatment to Bothrops genus envenomation is the administration of the specific anti-venom. If anti-venom administration is rapidly applied after envenomation, neutralization of systemic effects is usually successfully achieved. However, neutralization of local tissue damage is a more difficult task. In a number of snakebite cases, lack of neutralization of local effect results in permanent sequelae (Gutiérrez and Lomonte 1989; Rucavado and Lomonte, 1996; Wen, 2000).

Thus, new pharmacological approaches must be introduced to deal with those relevant effects.

The aim of this work was to investigate the efficacy of the extracts of Curcuma longa and Calendula officinalis on treatment of envenomation by Bothrops alternatus snake venom.

\section{MATERIAL AND METHODS}

The Calendula officinalis extract (ointment 10\%) and ar-turmerone (10\%) (Ferreira et al., 1992a) were obtained at the Institute of Chemistry, School of Veterinary Medicine, in Hannover, Germany. Rhizomes of Curcuma longa were collected from the botanical garden at the Universidade Federal de Minas Gerais, Brazil. Two extracts of Curcuma longa were utilized: $\left.1^{\circ}\right) 327$ grams of extracted in $100 \mathrm{ml}$ of distillated water plus $400 \mathrm{ml}$ of isopropanol, during $24 \mathrm{~h} ; 2^{\circ}$ ) 327 grams of extracted in $500 \mathrm{ml}$ of methanol during $24 \mathrm{~h}$.

Lyophilized venom of Bothrops alternatus snake was supplied by "Universidade de Alfenas" and stored at $-20^{\circ} \mathrm{C}$. The venom was dissolved in 
sterile physiological saline $(0.85 \% \quad \mathrm{w} / \mathrm{v} \mathrm{NaCl}$ solution), and injected intradermally $(1.25 \mu \mathrm{g} / 100 \mu \mathrm{l})$ into the shaved dorsal back skin of 30 New Zealand white rabbits (mean weight of $800 \mathrm{~g})$.

The rabbits were divided into six groups, with five animals each receiving the following treatments: group I - subcutaneous application with Curcuma longa extract $(1.0 \mathrm{ml})$; group II topic treatment of Curcuma longa hydroalcoholic extract $(1.0 \mathrm{ml})$; group III - topic application of ar-turmerone in vaseline $(1.0 \mathrm{~g})$; group IV - topic application of Curcuma longa methanolic extract $(1.0 \mathrm{ml})$; group $\mathrm{V}$ : topic application with Calendula officinalis ointment $(1.0 \mathrm{~g})$; group VI: topic application of saline $(1.0 \mathrm{ml})$. These treatments were done at 30 minutes, and at $2 \mathrm{~h}, 4 \mathrm{~h}, 24 \mathrm{~h}$ and $72 \mathrm{~h}$ after venom inoculation. After that, the animals were examined until 168 hours.

Edematogenic activity was estimated by pachymeter measuring at 30 minutes, $2 \mathrm{~h}, 4 \mathrm{~h}$, $24 \mathrm{~h}, 72 \mathrm{~h}, 96 \mathrm{~h}, 120 \mathrm{~h}, 144 \mathrm{~h}$ and $168 \mathrm{~h}$ after venom inoculation. The development of the hemorrhage was estimated by determination of the diameter of the lesion using a special millimeter ruler at the same times as described above and according to Ferreira et al. (1992b), with modifications (the evaluations were done outside of skin without excising it).

It was collected blood from the heart (EDTA $10 \%$ ) to process the hematological exams and blood without anti-coagulant to process seric biochemical parameters as follow: erythrocytes count (RBC); hemoglobin ( $\mathrm{Hb})$; packed volume cell (PVC); medium corpuscular value (MCV); medium corpuscular hemoglobin (MCH); medium corpuscular of hemoglobin concentration (MCHC); leukocytes count (WBC), relative count of lymphocytes, neutrophils, eosinophils, monocytes and bast cells; platelets count; total protein (TP); fibrinogen (Fibr); blood urea nitrogen (BUN) and creatinine (Creat) at 168 hours after venom injection (Ferreira Neto et al., 1982).

Necrosis and other lesions (edema, ischemia and inflammatory reaction) were evaluated in the skin by necropsy and histopathological exams. After 168 hours, all the animals were sacrificed by ether inhalation and were submitted to necropsy. Skin fragments $(1 \mathrm{~cm})$ were fixed into a $10 \%$ formalin buffered solution. After two hours, they were sliced to allow fixation. After incubation in formalin solution during 24 hours, the fragments were transferred to flasks containing different concentration of ethyl alcohol (70\%, 80\%, 90\% and 100\%) and maintained for two hours in each solution. Afterward, they were left until being embedded in paraffin, $5 \mu \mathrm{m}$ sectioned, and stained by hematoxilin/eosin (Luna, 1968).

The completely randomized design was employed. Mean and standard deviation error were calculated for hemorrhage diameter and edema within each group. The hematological and biochemical parameters were presented as the mean and statistical analysis was performed by Student's t-test, within the $95 \%$ of the level of significance.

\section{RESULTS}

The effects of extracts of plants and ar-turmerone fraction on hemorrhage, edema and necrosis evoked by Bothrops alternatus venom are shown on Tables 1, 2 and 3.

In group I (Curcuma longa subcutaneous), the overall mean of hemorrhagic halo was $22.94 \mathrm{~mm}$ and edema was $0.74 \mathrm{~mm}$. It is important to stress that the subcutaneous application of this extract did not prevent the subsequent hemorrhagic lesion development (Table 1). In the same group the necrosis point was observed $72 \mathrm{~h}$ after the venom inoculation in three animals.

In group II (Curcuma longa topic), the overall mean of hemorrhagic halo was $8.54 \mathrm{~mm}$ and edema was $0.61 \mathrm{~mm}$, similar to groups IV, V and VI. Four animals showed necrosis until $24 \mathrm{~h}$, and one rabbit until $168 \mathrm{~h}$ (Table 3 ). In this group, two animals died.

In Group III (ar-turmerone), the overall mean of hemorrhagic halo was $3.82 \mathrm{~mm}$ and edema was $0.31 \mathrm{~mm}$. Immediately after the first treatment, the hemorrhagic halo (Table 1) and edema (Table 2) decreased, and despite of mild necrosis occurrence, all the animals were cured within 96h (Table 3). 
Table 1. Mean values and standard error of means (SEM) of hemorrhage halo (in millimeter) after Bothrops alternatus envenomation and treatment of Curcuma longa, Calendula officinalis and arturmerone in different times

\begin{tabular}{|c|c|c|c|c|c|c|}
\hline Groups & $\mathrm{I}$ & II & III & IV & $\mathrm{V}$ & VI \\
\hline Time & $\begin{array}{c}\text { Curcuma longa } \\
\text { Subcutaneous }\end{array}$ & $\begin{array}{l}\text { Curcuma longa } \\
\text { Topic }\end{array}$ & Ar-turmerone & $\begin{array}{c}\text { Curcuma longa } \\
\text { Methanol }\end{array}$ & $\begin{array}{l}\text { Calendula } \\
\text { officinalis }\end{array}$ & Control \\
\hline $30 \mathrm{~min}$ & $\begin{array}{c}11.6 \mathrm{Aa} \\
\mathrm{SEM}=3.95\end{array}$ & $\begin{array}{c}25.2 \mathrm{Acb} \\
\mathrm{SEM}=3.95\end{array}$ & $\begin{array}{c}14.0 \mathrm{Aa} \\
\mathrm{SEM}=3.95\end{array}$ & $\begin{array}{c}29.8 \mathrm{Ab} \\
\mathrm{SEM}=3.95\end{array}$ & $\begin{array}{c}18.2 \mathrm{Aa} \\
\mathrm{SEM}=3.95\end{array}$ & $\begin{array}{c}15.2 \mathrm{Aac} \\
\mathrm{SEM}=3.95\end{array}$ \\
\hline $2 \mathrm{~h}$ & $\begin{array}{c}26.0 \mathrm{Ba} \\
\mathrm{SEM}=3.95\end{array}$ & $\begin{array}{c}12.0 \mathrm{Bb} \\
\mathrm{SEM}=3.95\end{array}$ & $\begin{array}{c}\text { 6.0Ab } \\
\mathrm{SEM}=3.95\end{array}$ & $\begin{array}{c}16.2 \mathrm{Ba} \\
\mathrm{SEM}=3.95\end{array}$ & $\begin{array}{c}16.8 \mathrm{Aa} \\
\mathrm{SEM}=3.95\end{array}$ & $\begin{array}{c}15.6 \mathrm{Aa} \\
\mathrm{SEM}=3.95\end{array}$ \\
\hline $4 h$ & $\begin{array}{c}30.8 \mathrm{BCa} \\
\mathrm{SEM}=3,95\end{array}$ & $\begin{array}{c}7.6 \mathrm{Bb} \\
\mathrm{SEM}=3.95\end{array}$ & $\begin{array}{c}5.2 \mathrm{Ab} \\
\mathrm{SEM}=3.95\end{array}$ & $\begin{array}{c}8.2 \mathrm{Bb} \\
\mathrm{SEM}=3.95\end{array}$ & $\begin{array}{c}17.0 \mathrm{Ab} \\
\mathrm{SEM}=3.95\end{array}$ & $\begin{array}{c}14.0 \mathrm{Ab} \\
\mathrm{SEM}=3.95\end{array}$ \\
\hline $24 \mathrm{~h}$ & $\begin{array}{c}35.0 \mathrm{BCa} \\
\mathrm{SEM}=3.95\end{array}$ & $\begin{array}{c}6.4 \mathrm{Bbc} \\
\mathrm{SEM}=3.95\end{array}$ & $\begin{array}{c}3.8 \mathrm{Ac} \\
\mathrm{SEM}=3.95\end{array}$ & $\begin{array}{c}\text { 7.6Bbc } \\
\mathrm{SEM}=3.95\end{array}$ & $\begin{array}{c}15.8 \mathrm{Ab} \\
\mathrm{SEM}=3.95\end{array}$ & $\begin{array}{c}11.8 \mathrm{Abc} \\
\mathrm{SEM}=3.95\end{array}$ \\
\hline $48 \mathrm{~h}$ & $\begin{array}{c}35.0 \mathrm{BCa} \\
\mathrm{SEM}=3.95\end{array}$ & $\begin{array}{c}6.0 \mathrm{Bbc} \\
\mathrm{SEM}=3.95\end{array}$ & $\begin{array}{c}3.8 \mathrm{Ac} \\
\mathrm{SEM}=3.95\end{array}$ & $\begin{array}{c}6.8 \mathrm{Bbc} \\
\mathrm{SEM}=3.95\end{array}$ & $\begin{array}{c}16.0 \mathrm{Ab} \\
\mathrm{SEM}=3.95\end{array}$ & $\begin{array}{c}12.6 \mathrm{Abc} \\
\mathrm{SEM}=3.95\end{array}$ \\
\hline $72 \mathrm{~h}$ & $\begin{array}{c}35.0 \mathrm{BCa} \\
\mathrm{SEM}=3.95\end{array}$ & $\begin{array}{c}5.8 \mathrm{Bb} \\
\mathrm{SEM}=3.95\end{array}$ & $\begin{array}{c}4.0 \mathrm{Ab} \\
\mathrm{SEM}=3.95\end{array}$ & $\begin{array}{c}6.8 \mathrm{Bb} \\
\mathrm{SEM}=3.95\end{array}$ & $\begin{array}{c}3.2 \mathrm{Bb} \\
\mathrm{SEM}=3.95\end{array}$ & $\begin{array}{c}12.4 \mathrm{Ab} \\
\mathrm{SEM}=3.95\end{array}$ \\
\hline $96 \mathrm{~h}$ & $\begin{array}{c}14.0 \mathrm{Aa} \\
\mathrm{SEM}=3.95\end{array}$ & $\begin{array}{c}4.0 \mathrm{Ba} \\
\mathrm{SEM}=4.42\end{array}$ & $\begin{array}{c}1.4 \mathrm{Bb} \\
\mathrm{SEM}=3.95\end{array}$ & $\begin{array}{c}1.6 \mathrm{Bb} \\
\mathrm{SEM}=3.95\end{array}$ & $\begin{array}{c}1.3 \mathrm{Bb} \\
\mathrm{SEM}=4.42\end{array}$ & $\begin{array}{c}3.2 \mathrm{Bab} \\
\mathrm{SEM}=3.95\end{array}$ \\
\hline $120 \mathrm{~h}$ & $\begin{array}{c}\text { 14.0Aba } \\
\mathrm{SEM}=3.95\end{array}$ & $\begin{array}{c}\text { 4.0Ba } \\
\mathrm{SEM}=3.95\end{array}$ & $\begin{array}{c}0 \mathrm{Bb} \\
\mathrm{SEM}=0\end{array}$ & $\begin{array}{c}1.8 \mathrm{Bb} \\
\mathrm{SEM}=3.95\end{array}$ & $\begin{array}{c}1.3 \mathrm{Bb} \\
\mathrm{SEM}=4.42\end{array}$ & $\begin{array}{c}3.0 \mathrm{Bb} \\
\mathrm{SEM}=3.95\end{array}$ \\
\hline $144 \mathrm{~h}$ & $\begin{array}{c}14.0 \mathrm{Aa} \\
\mathrm{SEM}=3.95\end{array}$ & $\begin{array}{c}\text { 4.0Ba } \\
\mathrm{SEM}=5.11\end{array}$ & $\begin{array}{c}0 \mathrm{Bb} \\
\mathrm{SEM}=0\end{array}$ & $\begin{array}{c}1.8 \mathrm{Bb} \\
\mathrm{SEM}=3.95\end{array}$ & $\begin{array}{c}1.5 \mathrm{Bb} \\
\mathrm{SEM}=4.42\end{array}$ & $\begin{array}{c}2.8 \mathrm{Bb} \\
\mathrm{SEM}=3.95\end{array}$ \\
\hline $168 \mathrm{~h}$ & $\begin{array}{c}14.0 \mathrm{Aa} \\
\mathrm{SEM}=3,95\end{array}$ & $\begin{array}{c}\text { 4.0Ba } \\
\mathrm{SEM}=3.95\end{array}$ & $\begin{array}{c}0 \mathrm{Bb} \\
\mathrm{SEM}=0\end{array}$ & $\begin{array}{c}1.8 \mathrm{Bb} \\
\mathrm{SEM}=3.95\end{array}$ & $\begin{array}{c}0.5 \mathrm{Bb} \\
\mathrm{SEM}=4.42\end{array}$ & $\begin{array}{c}2.8 \mathrm{Bb} \\
\mathrm{SEM}=3.95\end{array}$ \\
\hline Overall mean & 22.94 & 8.55 & 3.82 & 8.24 & 9.85 & 9.34 \\
\hline
\end{tabular}

In group IV (Curcuma longa in methanol), the overall mean of hemorrhagic halo was $8.24 \mathrm{~mm}$ and edema was $0.48 \mathrm{~mm}$, similar to groups II, V and VI. But in this group, all the animals $(n=5)$ showed mild necrosis points (Table 3). At 96h, four animals had already being cured, except one that still showed necrosis, diffuse hemorrhage and edema until $168 \mathrm{~h}$.

The Calendula officinalis ointment (group V) did not prevent hemorrhage, edema and necrosis formation, and the lesions were so severe like those observed in group VI (saline). In group V, the overall mean of hemorrhagic halo was $9.85 \mathrm{~mm}$ and edema was $0.64 \mathrm{~mm}$.

Animals from group VI (saline) showed edema (Table 2) and necrosis (Table 3) until 168h, and one animal was bleeding at the local of venom inoculation at $4 \mathrm{~h}$. The overall mean of hemorrhagic halo in group VI was $9.34 \mathrm{~mm}$ and edema was $0.70 \mathrm{~mm}$.

The results of treatment on hematological and seric biochemical alterations evoked by Bothrops alternatus venom are shown on Table 4. 
Table 2. Mean values and standard error of means (SEM) of edema (in millimeter) after Bothrops alternatus envenomation and treatment of Curcuma longa, Calendula officinalis and ar-turmerone in different times

\begin{tabular}{|c|c|c|c|c|c|c|}
\hline Groups & $\mathrm{I}$ & II & III & IV & $\mathrm{V}$ & VI \\
\hline Time & $\begin{array}{c}\text { Curcuma longa } \\
\text { Subcutaneous }\end{array}$ & $\begin{array}{c}\text { Curcuma longa } \\
\text { Topic }\end{array}$ & Ar-turmerone & $\begin{array}{c}\text { Curcuma longa } \\
\text { Methanol }\end{array}$ & $\begin{array}{l}\text { Calendula } \\
\text { officinalis }\end{array}$ & Control \\
\hline $30 \mathrm{~min}$ & $\begin{array}{c}0.70 \mathrm{Aa} \\
\mathrm{SEM}=0.15\end{array}$ & $\begin{array}{c}0.95 \mathrm{Aa} \\
\mathrm{SEM}=0.15\end{array}$ & $\begin{array}{c}0.78 \mathrm{Aa} \\
\mathrm{SEM}=0.15\end{array}$ & $\begin{array}{c}0.76 \mathrm{Aa} \\
\mathrm{SEM}=0.15\end{array}$ & $\begin{array}{c}0.72 \mathrm{ACa} \\
\mathrm{SEM}=0.15\end{array}$ & $\begin{array}{c}0.74 \mathrm{Aa} \\
\mathrm{SEM}=0.15\end{array}$ \\
\hline $2 \mathrm{~h}$ & $\begin{array}{c}1.28 \mathrm{Ba} \\
\mathrm{SEM}=0.15\end{array}$ & $\begin{array}{c}0.24 \mathrm{Bc} \\
\mathrm{SEM}=0.15\end{array}$ & $\begin{array}{c}0.34 \mathrm{BDbc} \\
\mathrm{SEM}=0.15\end{array}$ & $\begin{array}{c}0.70 \mathrm{Ab} \\
\mathrm{SEM}=0.15\end{array}$ & $\begin{array}{c}1.44 \mathrm{Ba} \\
\mathrm{SEM}=0.15\end{array}$ & $\begin{array}{c}1.32 \mathrm{Ba} \\
\mathrm{SEM}=0.15\end{array}$ \\
\hline $4 \mathrm{~h}$ & $\begin{array}{c}1.30 \mathrm{Ba} \\
\mathrm{SEM}=0.15\end{array}$ & $\begin{array}{c}0.70 \mathrm{Ab} \\
\mathrm{SEM}=0.15\end{array}$ & $\begin{array}{c}0.66 \mathrm{Ab} \\
\mathrm{SEM}=0.15\end{array}$ & $\begin{array}{c}0.64 \mathrm{Ab} \\
\mathrm{SEM}=0.15\end{array}$ & $\begin{array}{c}0.68 \mathrm{Ab} \\
\mathrm{SEM}=0.15\end{array}$ & $\begin{array}{c}0.98 \mathrm{Aab} \\
\mathrm{SEM}=0.15\end{array}$ \\
\hline $24 \mathrm{~h}$ & $\begin{array}{c}1.30 \mathrm{Ba} \\
\mathrm{SEM}=0.15\end{array}$ & $\begin{array}{c}1.32 \mathrm{Aa} \\
\mathrm{SEM}=0.15\end{array}$ & $\begin{array}{c}0.44 \mathrm{Ac} \\
\mathrm{SEM}=0.15\end{array}$ & $\begin{array}{c}1.00 \mathrm{Abc} \\
\mathrm{SEM}=0.15\end{array}$ & $\begin{array}{c}1.14 \mathrm{Bab} \\
\mathrm{SEM}=0.15\end{array}$ & $\begin{array}{c}1.44 \mathrm{Ba} \\
\mathrm{SEM}=0.15\end{array}$ \\
\hline $48 \mathrm{~h}$ & $\begin{array}{c}0.90 \mathrm{Aa} \\
\mathrm{SEM}=0.15\end{array}$ & $\begin{array}{c}0.58 \mathrm{Aab} \\
\mathrm{SEM}=0.15\end{array}$ & $\begin{array}{c}0.36 \mathrm{BCb} \\
\mathrm{SEM}=0.15\end{array}$ & $\begin{array}{c}0.72 \mathrm{Aa} \\
\mathrm{SEM}=0.15\end{array}$ & $\begin{array}{c}0.50 \mathrm{Aab} \\
\mathrm{SEM}=0.15\end{array}$ & $\begin{array}{c}0.84 \mathrm{Aa} \\
\mathrm{SEM}=0.15\end{array}$ \\
\hline $72 \mathrm{~h}$ & $\begin{array}{c}0.72 \mathrm{Aa} \\
\mathrm{SEM}=0.15\end{array}$ & $\begin{array}{c}0.70 \mathrm{Aa} \\
\mathrm{SEM}=0.15\end{array}$ & $\begin{array}{c}0.26 \mathrm{BCb} \\
\mathrm{SEM}=0.15\end{array}$ & $\begin{array}{c}0.64 \mathrm{Aba} \\
\mathrm{SEM}=0.15\end{array}$ & $\begin{array}{c}0.60 \mathrm{Aab} \\
\mathrm{SEM}=0.15\end{array}$ & $\begin{array}{c}0.70 \mathrm{Aa} \\
\mathrm{SEM}=0.15\end{array}$ \\
\hline $96 \mathrm{~h}$ & $\begin{array}{c}0.28 \mathrm{Da} \\
\mathrm{SEM}=0.15\end{array}$ & $\begin{array}{c}0.45 \mathrm{Ba} \\
\mathrm{SEM}=0.15\end{array}$ & $\begin{array}{c}0.06 \mathrm{Da} \\
\mathrm{SEM}=0.15\end{array}$ & $\begin{array}{c}0.16 \mathrm{Ba} \\
\mathrm{SEM}=0.15\end{array}$ & $\begin{array}{c}0.33 \mathrm{Aa} \\
\mathrm{SEM}=0.16\end{array}$ & $\begin{array}{c}0.36 \mathrm{Aa} \\
\mathrm{SEM}=0.15\end{array}$ \\
\hline $120 \mathrm{~h}$ & $\begin{array}{c}0.30 \mathrm{Da} \\
\mathrm{SEM}=0.15\end{array}$ & $\begin{array}{c}0.23 \mathrm{Ba} \\
\mathrm{SEM}=0.19\end{array}$ & $\begin{array}{c}0.06 \mathrm{Da} \\
\mathrm{SEM}=0.15\end{array}$ & $\begin{array}{c}0.08 \mathrm{Ba} \\
\mathrm{SEM}=0.15\end{array}$ & $\begin{array}{c}0.25 \mathrm{Ca} \\
\mathrm{SEM}=0.16\end{array}$ & $\begin{array}{c}0.22 \mathrm{Aa} \\
\mathrm{SEM}=0.15\end{array}$ \\
\hline $144 \mathrm{~h}$ & $\begin{array}{c}0.32 \mathrm{CDa} \\
\mathrm{SEM}=0.15\end{array}$ & $\begin{array}{c}0.23 \mathrm{Ba} \\
\mathrm{SEM}=0.19\end{array}$ & $\begin{array}{c}0.06 \mathrm{Da} \\
\mathrm{SEM}=0.15\end{array}$ & $\begin{array}{c}0.08 \mathrm{Ba} \\
\mathrm{SEM}=0.15\end{array}$ & $\begin{array}{c}0.25 \mathrm{Ca} \\
\mathrm{SEM}=0.16\end{array}$ & $\begin{array}{c}0.24 \mathrm{Aa} \\
\mathrm{SEM}=0.15\end{array}$ \\
\hline $168 \mathrm{~h}$ & $\begin{array}{c}0.32 \mathrm{CDa} \\
\mathrm{SEM}=0.15\end{array}$ & $\begin{array}{c}0.20 \mathrm{Ba} \\
\mathrm{SEM}=0.19\end{array}$ & $\begin{array}{c}0.06 \mathrm{Da} \\
\mathrm{SEM}=0.15\end{array}$ & $\begin{array}{c}0.06 \mathrm{Ba} \\
\mathrm{SEM}=0.15\end{array}$ & $\begin{array}{c}0.15 \mathrm{Ca} \\
\mathrm{SEM}=0.16\end{array}$ & $\begin{array}{c}0.18 \mathrm{Aa} \\
\mathrm{SEM}=0.15\end{array}$ \\
\hline Overall mean & 0.74 & 0.61 & 0.31 & 0.48 & 0.64 & 0.70 \\
\hline
\end{tabular}

Table 3. Presence of necrosis evoked by the Bothrops alternatus venom (mild, moderate and severe) after different treatments with Curcuma longa, Calendula officinalis and ar-turmerone

\begin{tabular}{|c|c|c|c|c|c|c|}
\hline Groups & $\mathrm{I}$ & II & III & IV & $\mathrm{V}$ & VI \\
\hline Time & $\begin{array}{c}\text { Curcuma longa } \\
\text { Subcutaneous }\end{array}$ & $\begin{array}{c}\text { Curcuma longa } \\
\text { Topic }\end{array}$ & Ar-turmerone & $\begin{array}{c}\text { Curcuma longa } \\
\text { Methanol }\end{array}$ & $\begin{array}{l}\text { Calendula } \\
\text { officinalis }\end{array}$ & Control \\
\hline $30 \mathrm{~min}$ & - & - & - & - & - & - \\
\hline $2 \mathrm{hr}$ & - & - & - & - & - & - \\
\hline $4 \mathrm{hr}$ & - & - & - & - & - & - \\
\hline $24 \mathrm{hr}$ & - & $+(1 / 5)$ & $+(1 / 5)$ & $+(2 / 5)$ & $+(1 / 5)$ & $+(2 / 5)$ \\
\hline $48 \mathrm{hr}$ & - & $++(1 / 5)$ & $+(3 / 5)$ & $+(4 / 5)$ & $+++(4 / 5)$ & $+++(4 / 5)$ \\
\hline $72 \mathrm{hr}$ & $+(3 / 5)$ & $++(1 / 5)$ & $+(2 / 5)$ & $+(5 / 5)$ & $+++(4 / 5)$ & $+++(4 / 5)$ \\
\hline $96 \mathrm{hr}$ & $+(1 / 5)$ & $++(1 / 4)$ & - & $+(1 / 5)$ & $+++(2 / 4)$ & $+++(3 / 5)$ \\
\hline $120 \mathrm{hr}$ & $+(1 / 5)$ & $++(1 / 3)$ & - & $+(1 / 5)$ & $+++(2 / 4)$ & $+++(3 / 5)$ \\
\hline $144 \mathrm{hr}$ & $+(1 / 5)$ & $++(1 / 3)$ & - & $+(1 / 5)$ & $+++(2 / 4)$ & $+++(3 / 5)$ \\
\hline $168 \mathrm{hr}$ & $+(1 / 5)$ & $++(1 / 3)$ & - & $+(1 / 5)$ & $+++(1 / 4)$ & $+++(3 / 5)$ \\
\hline
\end{tabular}


Table 4. Hematological and serum biochemical values (mean values) of rabbits envenomed by Bothrops alternatus after different treatments

\begin{tabular}{lcccccc}
\hline Groups & I & II & III & IV & V & VI \\
\hline RBC/mm3 & $4,022000 \mathrm{~B}$ & $6,086000 \mathrm{BA}$ & $5,504000 \mathrm{BA}$ & $5,666000 \mathrm{BA}$ & $6,988000 \mathrm{~A}$ & $5,332000 \mathrm{BA}$ \\
$\mathrm{Hb}$ g/dl & $8.5 \mathrm{~A}$ & $12.1 \mathrm{~A}$ & $10.9 \mathrm{~A}$ & $10.4 \mathrm{~A}$ & $11.8 \mathrm{~A}$ & $10.4 \mathrm{~A}$ \\
PCV \% & $28.4 \mathrm{~A}$ & $40.6 \mathrm{~A}$ & $35.4 \mathrm{~A}$ & $34.8 \mathrm{~A}$ & $39.0 \mathrm{~A}$ & $35.2 \mathrm{~A}$ \\
$\mathrm{MCV} \mathrm{fl}$ & $75.9 \mathrm{~A}$ & $67.9 \mathrm{BA}$ & $64.9 \mathrm{BA}$ & $61.2 \mathrm{~B}$ & $57.7 \mathrm{~B}$ & $66.5 \mathrm{~B}$ \\
$\mathrm{MCH} \mathrm{pg}$ & $21.6 \mathrm{~A}$ & $20.2 \mathrm{BA}$ & $19.8 \mathrm{BA}$ & $18.3 \mathrm{~B}$ & $17.6 \mathrm{~B}$ & $19.6 \mathrm{BA}$ \\
MCHC \% & $28.4 \mathrm{~A}$ & $29.8 \mathrm{~A}$ & $30.1 \mathrm{~A}$ & $29.9 \mathrm{~A}$ & $30.4 \mathrm{~A}$ & $29.5 \mathrm{~A}$ \\
TP mg/dl & $4.1 \mathrm{~A}$ & $5.8 \mathrm{~A}$ & $5.7 \mathrm{~A}$ & $5.9 \mathrm{~A}$ & $5.2 \mathrm{~A}$ & $5.9 \mathrm{~A}$ \\
Fibr mg/dl & $3.4 \mathrm{~A}$ & $4,9 \mathrm{~A}$ & $5,6 \mathrm{~A}$ & $6,6 \mathrm{~A}$ & $4,7 \mathrm{~A}$ & $7,3 \mathrm{~A}$ \\
WBC/mm3 & $4,844 \mathrm{~A}$ & $6,956 \mathrm{~A}$ & $4,780 \mathrm{~A}$ & $8,316 \mathrm{~A}$ & $7,692 \mathrm{~A}$ & $8,594 \mathrm{~A}$ \\
Lymphocyte $\%$ & $52.8 \mathrm{~A}$ & $50.0 \mathrm{~A}$ & $37.2 \mathrm{~A}$ & $43.6 \mathrm{~A}$ & $45.6 \mathrm{~A}$ & $42.4 \mathrm{~A}$ \\
Neutrophil \% & $45.6 \mathrm{~A}$ & $46.0 \mathrm{~A}$ & $59.2 \mathrm{~A}$ & $53.6 \mathrm{~A}$ & $51.6 \mathrm{~A}$ & $52.0 \mathrm{~A}$ \\
Eosinophil \% & $0 \mathrm{~A}$ & $0 \mathrm{~A}$ & $0 \mathrm{~A}$ & $0 \mathrm{~A}$ & $0 \mathrm{~A}$ & $0.4 \mathrm{~A}$ \\
Monocyte \% & $0.4 \mathrm{~A}$ & $4.6 \mathrm{~B}$ & $3.6 \mathrm{BA}$ & $2.8 \mathrm{BA}$ & $2.4 \mathrm{BA}$ & $4.4 \mathrm{~A}$ \\
Bast cell \% & $0 \mathrm{~A}$ & $0 \mathrm{~A}$ & $0 \mathrm{~A}$ & $0 \mathrm{~A}$ & $0 \mathrm{~A}$ & $0.8 \mathrm{~A}$ \\
Platelets/mm3 & $157.6 \mathrm{C}$ & $272.7 \mathrm{BAC}$ & $198.5 \mathrm{BC}$ & $428.3 \mathrm{~A}$ & $406.6 \mathrm{BA}$ & $182.1 \mathrm{C}$ \\
BUN mg/dl & $44.2 \mathrm{~A}$ & $30.2 \mathrm{BA}$ & $40.63 \mathrm{BA}$ & $17.3 \mathrm{~B}$ & $20.1 \mathrm{BA}$ & $32.8 \mathrm{BA}$ \\
Creat mg/dl & $1.17 \mathrm{~A}$ & $1.03 \mathrm{~A}$ & $0.95 \mathrm{~A}$ & $0.85 \mathrm{~A}$ & $0.74 \mathrm{~A}$ & $0.95 \mathrm{~A}$ \\
\hline
\end{tabular}

Different letters in the same row indicate a statistically difference $(\mathrm{P} \leq 0.05)$.

Results from group I (Table 4) showed smallest values of erythrocytes, hemoglobin and packed corpuscular volume (PCV). Maybe, the hematological results in group I are due to the subcutaneous treatment, that causes more hemorrhage. Further, total protein, fibrinogen and platelets results were decreased.

Groups IV and VI (control) showed leukocytosis with eosinopenia and only in group VI bast cells were seen.

Kidney failure was not observed; all animals showed normal levels of creatinine and BUN

In group I, the microscopic findings on skin (inoculation area) after Bothrops alternatus envenomation were edema presence and hemorrhage in dermis deeper layers, plasmocytes in degeneration process and cell debris besides extensive hemorrhagic areas and dilatation vessels. In subcutaneous tissue, it was seen amorphous substance of basophilic aspect. Many hair follicles showed hemorrhagic and necrotic changes. Muscular layer of subcutaneous tissue showed inflammatory reaction, with mononuclear cells (plasmocytes and lymphocytes), besides edema and hemorrhage. In some areas, it was observed severe inflammatory reaction, constituted by degenerated polymorphonuclear neutrophils (pyocytes) and other areas showed hemorrhage, coagulation necrosis and edema. In group II, the dermis showed edema, hemorrhage and inflammatory infiltrated (macrophages) with degenerated cells and cells debris. The dermis was characterized by extensive areas of necrosis and dilatation of lymphatic vessels. In group III, the dermis showed mild inflammatory reaction constituted by eosinophils, besides mild edema and dilated hair follicles. The group IV showed mild edema in dermis without hemorrhage and some areas of necrosis and presence of inflammatory reaction, with mononuclear cells (macrophages and lymphocytes). The epidermis was characterized by parakeratosis (retention of nuclei in the keratin layer with the absence of the granular layer). The group $\mathrm{V}$ showed ulcerative dermatitis and epidermis was completely degenerated with severe inflammatory reaction (neutrophils, lymphocytes and macrophages) and necrosis. In group VI, the epidermis was completely degenerated too. The hair follicles were dilated with amorphous substance (keratin retention). In addition, dermis had severe inflammatory reaction (neutrophils, lymphocytes and macrophages) with necrosis, besides hemorrhage and hemossiderin.

\section{DISCUSSION}

Two hours after the venom inoculation only group I (subcutaneous application of Curcuma 
longa) showed increased hemorrhagic halo diameter, bigger than control group (application of saline) (Table 1). Theses results show that the parenteral treatments (subcutaneous, for example) in Bothrops envenomation must be excluded due to the metalloproteinase present in this venom, which causes degradation of endothelial vessels, besides the presence of substances that causes coagulation disorders (Kamiguti et al., 1998). Further, skin necrosis was observed in three animals.

Calendula officinalis ointment must not be used in local treatment of Bothrops alternatus envenomation in accordance to Melo et al. (2000). Besides this treatment does not prevent the local hemorrhage, it also does not prevent local edema and necrosis (that was present in four animals). Until 48h, there was no statistically significant difference between this treatment and control group.

The aqueous extract of topic Curcuma longa (group II) showed unsatisfactory effect to recover edema (Table 2), because there was no statistically significant difference between it and group I (control). Furthermore, in this group, four animals showed necrosis at different times, and two animals died.

So, subcutaneous and topically treatments of Curcuma longa and Calendula officinalis ointment did not prevent the necrosis in $B$. alternatus envenomation.

The animals treated with ar-turmerone (group III) presented the best results (Table 2): evident decrease of hemorrhagic halo in skin $(\mathrm{P} \leq 0.05)$, smaller edema degree $(\mathrm{P} \leq 0.05)$ and completely recover (necrosis absence) at $96 \mathrm{~h}$ (Table 3 ).

The rabbits from group IV (Curcuma longa in methanol) did not show good results in first $2 \mathrm{~h}$, regarding to recovering of hemorrhagic halo and edema. But at 4 and $24 \mathrm{~h}$, there was no statistically difference $(\mathrm{P}>0.05)$ between that and control group. All the animals from this group showed necrosis points and one showed pain, necrosis, diffuse hemorrhage and edema until $168 \mathrm{~h}$. Thus, the physicians must avoid the local treatments with alcoholic extracts (methanolic tincture without evaporation) in injured skin.
The local necrotizing effect often results in the rapid development of a frequently severe local lesion (Rosenfeld, 1971), and the treatments with specific antivenoms does not appear to be able of neutralizing this venom-induced local inflammation (Gutiérrez et al., 1986; Gutiérrez and Lomonte, 1989; Wen, 2000).

It is known that the hemorrhage toxins present in the venom act directly on the capillary basement membrane and on the endothelial cells to cause internal hemorrhage and edema (Gutiérrez and Lomonte, 1989). This damage can activate cellular effects to release endogenous mediators of inflammation. Mediators of the inflammatory reaction are involved after Bothrops jararaca venom inoculation. Among them, products of arachidonic acid metabolism formed by the action of cycloxygenase and lipoxygenase, particularly leukotriene $\mathrm{B}_{4}$ and thromboxane $\mathrm{A}_{2}$ (Farsky et al., 1997).

Flores et al. (1993) reported that arachidonatederived lipoxygenase metabolites, such as leukotriene $\mathrm{B}_{4}$, act as chemotactic mediator after Bothrops erythromelas and Bothrops alternatus envenomation.

In a pharmacological study of edema induced by Bothrops asper venom, Chaves et al. (1995) reported that drugs like prazosin and indomethacin, administrated after venom injection, were effective in reducing edema, and theses results suggested that this edema is partially mediated by metalloproteinases, phospholipase $\mathrm{A}_{2}$, eicosanoids products and activation of $1 \alpha$ and $2 \beta$ adrenergic reptors.

Moura-da-Silva (1996) suggests that endogenous mechanisms of inflammatory response can be actived by viper venom metalloproteinases (like jararhagin) and supports the hypothesis that jararhagin can act as a pro-inflammatory stimulus, by inducing the production of cytokines by inflammatory cells (Moura-daSilva, 2001).

In the case of Bothrops envenomation, local edema is a common finding, playing a significant role in hypovolemia and being responsible for increments in intracompartmental pressure. Metalloproteinases and phospholipases A2 are abundant in Bothrops spp. venom and can induce edema (Lomonte and Gutiérrez, 1989). It is 
suggested that phospholipases $\mathrm{A}_{2}$ induce edema by two different mechanisms: (a) by releasing arachidonic acid as a consequence of enzymatic degradation of membrane phospholipids, enhancing the biosynthesis of eicosanoids, and (b) by directly affecting the microvasculatura, thereby causing plasmatic exsudation (Chaves et al., 1995).

Experimental studies indicate that the commercially available polyvalent antivenom is only partially effective in the neutralization of this activity (Gutiérrez et al., 1981, 1986).

Many researches are trying to find the best and efficient treatment capable of neutralizing the local inflammation induced by Bothrops venom. Three diarylheptanoids, curcumin 15 (diferuylmethane), demethoxycurcumin and bis demethoxycurcumin, make up the yellow dye of rhizomes of turmeric, Curcuma longa. These curcuminoids are the only natural pigments of this class. Well known as a spice and coloring matter, turmeric is also widely used as medicinal in oriental tradition, the treatment of snake bite poisoning being one its use. In the structure of curcumin, two ferulic acid moieties are linked via a methylene bridge, resulting in a conjugated diketone, which, in solution, through keto-enol tautomerism, produces a strong chelating centre. Many elements have been shown to form chelates with the curcuminoids. There are indications that curcumin also interacts strongly with biological macromolecules, like serum proteins, albumin and hyaluronic acid (Mors et al., 2000). Among the many pharmacological properties shown by curcuminoids, antiinflammatory, hepatoprotective, inhibition of lipoxygenase and prostaglandin-endoperoxide synthetase stand out (Kiso et al., 1983; Amman and Wahl, 1990).

Another unsaturated ketones of turmeric, arturmerone, neutralize both the hemorrhagic activities present in Bothrops jararaca venom, and lethal effect of Crotalus durissus terrificus venom in mice (Ferreira et al., 1992a).

Ar-turmerone has satisfactory effect to recover spot lesions being, until now, the best substance. But, at the present moment, it is unknown how the ar-turmerone acts exactly. The ar-turmerone may act as an enzymatic inhibitor in case of venom enzymes of $B$. alternatus with proteolytic and hemorrhagic activities, or may possess a direct antitoxin action or chemical inactivation, or inhibits the cyclogenase pathway (ecosainod products). It cannot be excluded, however, that it has been involved in immunological mechanisms, because Ferreira et al. (1992a) showed that extracts from Curcuma longa inhibited the proliferative response of human lymphocytes to PHA.

Calendula officinalis has flavonoids (isorhamnetin) in its flowers (which are the reputed active part of the plant). The flavonoids show the structural feature of the proximity and coplanarity of the phenolic hydroxyl group on the carbon atom 5 and the pyronic carbonyl (Mors et al., 2000). Moreover, Lindahl and Tagesson (1997) described the inhibitory effect of flavonoids on phospholipase from several species of snakes. But in this experiment, the treatment with Calendula officinalis ointment in Bothrops alternatus envenomation was not good.

The values of total erythrocytes (RBC), hemoglobin level $(\mathrm{Hb})$ and packet volume (PVC) cell were decreased in group I, once compared among all groups, including control group. These alterations can be explained by successive treatments (subcutaneous), which cause hemorrhage. These results are similar to Takahira (1996), that reported decrease in RBC, $\mathrm{Hb}$ and VPC, granting also the successive blood collects done in that experiment.

The values of leukocyte count (WBC) were decreased in group III (ar-turmerone fraction) compared to those obtained from group VI (control). Probably, ar-turmerone has antiinflammatory properties.

The neutrophils were greater in number in groups which presented the highest necrosis incidence. The inflammatory reaction that occurred in local inoculation of bothropic venom justifies this result, also reported by SanoMartins (1995) and Takahira (1996).

The platelets represent an important hemostatic role and for this reason they need to be numerally as functionally normal (Troy, 1988; Jain, 1993; Thomazini and Barraviera, 1994). The platelets were smaller in number in group II, which showed higher values of hemorrhagic halo, possibly due to the subcutaneous treatment 
that causes consequently great capillary fragility. It is described the venom capacity to cause thrombocytopenia, that can be consequent of alterations on a platelets function, besides metalloproteinases presence that cause vascular lesion causing platelets consume (Kamiguti et al., 1992, 1996).

The low plasmatic fibrinogen levels, resulting of thrombin-like enzyme from Bothrops venom described by Nahas et al. (1979), can be specially observed in group I. Systemic and local bleeding are complications commonly found in viperine envenoming. In Bothrops venoms, which also possess procoagulants, bleeding may be particularly severe, because fibrinogen consumption resulting incoagulable blood may coexist with thrombocytopenia.

The results of blood urea (BUN) and creatinine (creat) did not show alteration on kidney function among all groups. Despite Barraviera and Pereira (1994) claimed this as a rare complication of snake accident, this symptom could not have been observed, due to the quantity of venom inoculated.

The histopathological results agree with these clinical results, revealing, so far, that topic application of ar-turmerone showed the best results against the local effects of the venom from the Bothrops alternatus. Ferreira et al. (1992) reported that ar-turmerone neutralizes the hemorrhagic activity present in Bothrops jararaca venom and Gutiérrez et al. (1984) suggested that the main cause of poor regeneration is the decrease in blood supply to regenerating muscle. But in other research, Gutiérrez et al. (1986) reported that the elimination of hemorrhage did not result in complete regeneration in their experimental condition. These results showed that there is a need for more comprehensive studies on skeletal muscle regeneration after venom inoculations.

It is important to stress that subcutaneous application of Curcuma longa extract did not work, because this extract was not absorbed, resulting in amorphous substance in subcutaneous tissue.

In spite of the Calendula officinalis has an antiinflammatory substance (Mors et al., 2000), group $\mathrm{V}$, treated with this ointment, showed bad result, similar to that from group VI (saline): skin ulcerative lesion.

There were two classifications to myonecrosis in bothropic envenomation: myolytic vs coagulative. Queiroz and Petta (1984) when worked with Bothrops alternatus venom, described hyaline necrosis of media in arteries of muscle tissue in mice and the authors suggested there are, at least, two different ways by which Bothrops venom affect muscle cells: (a) directly, by action of miotoxins which probably affect the integrity of skeletal muscle plasma membrane; (b) indirectly, through an ischemic condition that develops in skeletal muscle secondarily to the disruptive action of venoms on the vasculature.

Queiroz et al. (1985) inoculated crude $B$. jararaca venom $(20 \mu \mathrm{g})$ and reported haemorrhage, myonecrosis and arterial necrosis. Myonecrosis was still observed with lower doses lower than $5 \mu \mathrm{g}$ of crude venom. Selistre et al. (1990) reported that Bothrops insularis venom $(20 \mu \mathrm{g}), 2$ hours after inoculation, caused extensive hemorrhage, myonecrosis, blood vessel necrosis and thrombosis, in paraffin sections of whole limb.

The gravidity of local and systemic harmful effect, which occurs in the bothropic envenomation, seems to be due to a synergistic action of several venom components.

\section{CONCLUSIONS}

Until now, the best local treatment against edema, necrosis and local hemorrhage after Bothrops alternatus envenomation is the arturmerone. Therefore, studies are underway in order to identify other substances to local treatment in Bothrops envenomation.

\section{REFERENCES}

AMMAN, H.P.T.; WAHL, M.A. Pharmacology of Curcuma longa. Planta Medica, v.57, p.1-7, 1990.

BARRAVIERA, B.; PEREIRA, P.C.M. Acidentes por serpentes do gênero Bothrops. In: BARRAVIERA, B. (Coord.) Venenos animais: uma visão integrada. Rio de Janeiro: Publicações Científicas, 1994. Cap.19, p.261-280. 
CHAVES, F.; BARBOZA, M.; GUTIÉRREZ, J.M. Pharmacological study of edema induced by venom of snake Bothrops asper (terciopelo) in mice. Toxicon, v.33, p.31-39, 1995.

FARSKY, S.H.P.; COSTA-CRUZ-J.W.M.; CURRY, Y. et al. Leuckocyte response induced by Bothrops jararaca crude venom: in vivo and in vitro studies. Toxicon, v.35, p.185-193, 1997.

FERREIRA, L.A.; HENRIQUES, O.B.; ANDREONI, A.A.S. et al. Antivenom and biological effects of ar-turmerone isolated from Curcuma longa (Zingiberaceae). Toxicon, v.30, p.1211-1218, 1992a.

FERREIRA, M.L.; MOURA-DA-SILVA, A.M.; FRANCCA, F.O.S. et al. Toxic activities of venoms from nine Bothrops species and their correlacion with lethality and necrosis. Toxicon, v.30, p.1603$1608,1992 b$.

FERREIRA-NETO, J.M.; VIANA, E.S.; MAGALHÃES, L.M. Patologia clínica veterinária. 2.ed. Belo Horizonte: Rabelo, 1982. 293p.

FLORES, C.A.; ZAPPELLINI, A.; PRADOFRANCESCHI, J. Lipoxygenase-derived mediators may be involved in in vitro neutrophil migration induced by Bothrops erythromelas and Bothrops alternatus venoms. Toxicon, v.31, p.15511559,1993 .

GUTIÉRREZ, J.M.; CHAVES, F.; BOLANOS, R. et al. Neutralización de los efectos locales del veneno de Bothrops asper por um antiveneno polivalente. Toxicon, v.19, p.493-500, 1981.

GUTIÉRREZ, J.M.; CHAVES, F.; MATA, E. et al. Skeletal muscle regeneration after myonecrosis innduced by Bothrops asper (Terciopelo) venom. Toxicon, v.24, p.223-231, 1986.

GUTIÉRREZ, J.M.; LOMONTE, B. Local tissue damage induced by Bothrops snake venoms. A Review. Mem. Inst. Butantan, v.51, p.211-223, 1989.

GUTIÉRREZ, J.M.; OWNBY, C.L.; ODELL, G.V. Skeletal muscle regeneration after myonecrosis induced by crude venom and a myotoxin from the snake Bothrops asper (Fer-de-Lance) venom. Toxicon, v.22, p.719-731, 1984.

GUTIÉRREZ, J.M.; ROJAS, G.; LOMONTE, B. et al. Comparative study of the edema-forming activity of Costa Rica snake venoms and its neutralization by a polyvalent antivenom. Comp. Bioch. Physiol., v.85C, p.175-176, 1986.

JAIN, N.C. Essentials of veterinary hematology. Philadelphia: Lea \& Febiger, 1993. 417p.
KAMIGUTI, A.S.; HAY, C.RM.; THEAKSTON, R.D.G. et al. Review Article. Insights into the mechanism of haemorrhage caused by snake venom metalloproteinases. Toxicon, v.34, p.327-642, 1996.

KAMIGUTI, A.S.; RUGMAN, F.P.; THEAKSTON, R.D.G. et al. The role of venom haemorrhagin in spontaneous bleeding in Bothrops jararaca envenoming. Thromb. Haemost., v.67, p.484-488, 1992.

KISO, Y.; SUZUKI, Y.; WATANABE, N. et al. Antihepatotoxic principles of Curcuma longa rhizomes. Planta Medica, v.49, p.185-187, 1983.

LINDAHL M.; TAGESSON, C. Flavonoids as phospholipases A2 inhibitors: importance of their structure for selective inhibition of group II phospholipases A2. Inflammation, v.21, p.347-356, 1997.

LOMONTE, B.; GUTIÉRREZ, J.M. A new muscle damaging toxin, myotoxin II, from the venom of the snake Bothrops asper (terciopelo). Toxicon, v.27, p.725-733, 1989.

LUNA, L.G. Manual of histologic staining methods of the Armed Forces Institute of Pathology. 3.ed. New York: McGraw Hill, 1968.

MELO, M.M.; HABERMEHL, G.G.; OLIVEIRA, N.J.F. Drugs against snake bite. SIMPÓSIO DA SOCIEDADE BRASILEIRA DE TOXINOLOGIA, 6., São Pedro, SP. Anais... SBT, 2000. P.130.

MORS W.B.; NASCIMENTO, M.C.; PEREIRA, B.M.R. et al. Plant natural products active against snake bite - the molecular approach. Phytochesmitry, v.55, p.627-642, 2000.

MOURA-DA-SILVA, A.M.; LAING, G.D; PAINE, M.J.L. et al. Processing of pro-tumour necrosis factor by venom metalloproteinases: a hipothesis explaining local tissue damage following snake bite. Europ. J. Immunol., v.26, p.2000-2005, 1996.

MOURA-DA-SILVA, A.M.; MARCINKIEWICZ, C.; MARCINKIEWICZ, M. et al. Selective recognition of $\alpha_{2} \beta_{1}$ integrin by jararhagin, a metalloproteinase/disintegrin from Bothrops jararaca venom. Thromb. Res., v.102, p.153-159, 2001.

NAHAS, L.; KAMIGUTI, A.S.; BARROS, M.A.R. Thrombin-like and fator X-activator components of Bothrops snake venoms. Thromb. Haemost., v.41, p.314-328, 1979.

QUEIROZ, L.S.; PETTA, C.A. Histopatological changes caused by urutu snake (Bothrops 
alternatus) in mouse skeletal muscle. Rev. Inst. Med. Trop. São Paulo, v.26, p.247-253, 1984.

QUEIROZ, L.S.; SANTO NETO, H.; ASSAKURA, M.T. et al. Patlological changes in muscle caused by haemorrhagic and proteolytic factors from Bothrops jararaca snake venom. Toxicon, v.23, p.341-345, 1985.

ROSENFELD, G. Symptomatology, pathology and treatment of snake bites in South America. In: BUCHERL, W.; BUCKLEY, E.E. (Eds.). Venomous animals and their venoms, v.2. New York: Academic, 1971. P.345-362.

RUCAVADO A.; LOMONTE, B. Neutralization of mionecrosis, hemorrhage, and edema induced by Bothrops asper snake venom by homologous and heterologous pre-existing antibodies in mice. Toxicon, v.34, p.567-577, 1996.

SANO-MARTINS, I.S.; SANTORO, M.L.; MORENA, P. et al. Hematological changes induced by Bothrops jararaca venom in dogs. Braz. J. Med. Biol. Res., v.28, p.303-312, 1995.

SELISTRE, H.S.; QUEIROZ, L.S.; CUNHA, O.A.B. et al. Isolation and characterization of hemorrhagic, myonecrotic and edema-inducing toxins from Bothrops insularis (jararaca ilhoa) snake venom. Toxicon, v.28, p.261-273, 1990.

TAKAHIRA, R. Alterações hemostáticas, hematológicas e bioquímicas de cães no envenenamento experimental por Bothrops jararaca (Wied, 1824) e Bothrops newiedi (Wangler, 1824). 1996. 177p. Dissertação (Mestrado) - Faculdade de Medicina Veterinária e Zootecnia, Universidade Estadual Paulista, Botucatu, SP.

THOMAZINI, I.A.; BARRAVIERA, B. Alterações hematológicas nos acidentes por animais peçonhentos. In: BARRAVIERA, B. (Coord.) Venenos animais: uma visão integrada. Rio de Janeiro: Publicações Científicas, 1994. Cap.6, p.8196.

TROY, G.G. An overview of hemostasis. Vet. Clin. North Am.: Small Anim. Pract., v.18, p.5-20, 1988.

WEN, F.H. Ineficácia do antiveneno na reversão do edema e necrose em pacientes picados por serpentes do gênero Bothrops. SIMPÓSIO DA SOCIEDADE BRASILEIRA DE TOXINOLOGIA, 6., São Pedro, SP. Anais... SBT, 2000. P.78-81. 